A modified two-slit interferometer for characterizing the asymmetric lateral coherence of undulator radiation

This content has been downloaded from IOPscience. Please scroll down to see the full text. 2016 EPL 11514004

(http://iopscience.iop.org/0295-5075/115/1/14004)

View the table of contents for this issue, or go to the journal homepage for more

Download details:

IP Address: 159.149.193.149

This content was downloaded on 06/03/2017 at 11:36

Please note that terms and conditions apply.

You may also be interested in:

Asymmetric lateral coherence of betatron radiation emitted in laser-driven light sources

B. Paroli, E. Chiadroni, M. Ferrario et al.

Propagation of transverse coherence

Makina Yabashi, Kenji Tamasaku, Shunji Goto et al.

Two-dimensional electromagnetically induced grating in coherent atomic medium

Yuyuan Chen, Zhuanzhuan Liu and Rengang Wan

Cold electron sources using laser-cooled atoms

Andrew J McCulloch, Ben M Sparkes and Robert E Scholten

Undulator and laser sources of soft $x$ rays

Evgenii G Bessonov and Aleksandr V Vinogradov

Determination of diffusion tensors from oscillating and circulating scanning probe tips

Susanne Hahne, Philipp Rahe and Philipp Maass

Far-field super-resolution imaging with a planar hyperbolic metamaterial lens

Cheng Lv, Wei Li, Xunya Jiang et al.

$\underline{\text { Reciprocating motion of active deformable particles }}$

M. Tarama and T. Ohta

Origin of emission from square-shaped organic microlasers

S. Bittner, C. Lafargue, I. Gozhyk et al. 


\title{
A modified two-slit interferometer for characterizing the asymmetric lateral coherence of undulator radiation
}

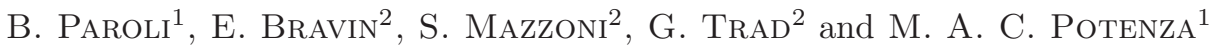 \\ 1 Dipartimento di Fisica, Universitá degli Studi di Milano and INFN Sezione di Milano - via G. Celoria, 16, \\ 20133 Milano, Italy \\ ${ }^{2}$ CERN - CH-1211 Geneva 23, Switzerland
}

received 13 May 2016; accepted in final form 13 July 2016

published online 8 August 2016

PACS 42.25.Kb - Coherence

PACS 41.60.Ap - Synchrotron radiation

PACS 41.75. Jv - Laser-driven acceleration

\begin{abstract}
Recently we have shown that the asymmetric lateral coherence of betatron radiation is characterized by peculiar properties that are evidenced with the analysis of the coherence factor of radiation. We extend such results to radiation emitted by ultra-relativistic proton beams in a 2-periods undulator. Results of a 2-dimensional simulation show that the real analysis of the asymmetric lateral coherence substantially improves the resolving power, thus transverse beam non-uniformities and the beam size can be measured at large distance.
\end{abstract}

Copyright (C) EPLA, 2016

Introduction. - The diagnostics of radiation emitted in large-scale accelerators (e.g., synchrotrons) or in more compact devices (as in laser-plasma-based accelerators) is fundamental to know the transverse properties of the particle beam source [1]. In particular the transverse beam size can be indirectly deduced by means of a measurement of the spatial transverse coherence of radiation when the condition of quasi-homogeneous source is satisfied as a consequence of the Van Cittert-Zernike theorem [2]. It can be measured, for example, with interferometric methods reminiscent of the double-slit Young interferometer [3], by diffraction of radiation from a single slit or a fiber [4] or by the analysis of the speckle field produced by diffraction of an ensemble of small scatterers $[5,6]$. Intensity interferometry can be also properly used to get information on both the transverse coherence and the particle bunch length $[7,8]$. A different approach is the imaging of the beam source by means of refractive lenses or small apertures $[9,10]$. In this case the diffraction limit gives an ultimate resolving power $\approx 1.22 \mathrm{z} \lambda / \mathrm{D}$ [11], where $z$ is the detection distance from the source and $D$ is the diameter of the lens or aperture (here a unit magnification and focal length $f=z / 2$ are considered). Recently we have shown that several source parameters can be deduced exploiting an asymmetric detection method of betatron radiation emitted by laser-plasma-accelerated particles $[12-14]$. The key quantity that contains information about the transverse coherence properties is the modulus of the coherence factor defined as

$$
\gamma_{c}(\tau)=\frac{\Gamma\left(\vec{X} ; \vec{X}_{0} ; \tau\right)}{\left[\Gamma\left(\vec{X}_{0} ; \vec{X}_{0} ; 0\right) \Gamma(\vec{X} ; \vec{X} ; 0)\right]^{1 / 2}}
$$

where

$$
\Gamma\left(\vec{X} ; \vec{X}_{0} ; \tau\right)=\lim _{T \rightarrow \infty} \frac{1}{2 T} \int_{-T}^{T} E(\vec{X}, t+\tau) E^{*}\left(\vec{X}_{0}, t\right) \mathrm{d} t
$$

is the cross-correlation function of the electric field $E$ (x-component) defined in a 2-dimensional reference frame. The fixed point $\vec{X}_{0}=\left(x_{0}, z_{0}\right)$ of eq. (1) plays an important role. When it is far from the average axis of motion $z$, the modulus of the coherence factor shows some multiple oscillations related to several source parameters. More in detail the off-axis position of $\vec{X}_{0}$ should be such that at least the weaker condition $\Delta L \approx L_{c}$ must be satisfied, where $\Delta L=L-L_{0}+\left(t-t_{0}\right) c$ is the difference between two optical paths along $\vec{\beta}$, from the source trajectory to the detector and $L_{c} \approx c \tau_{c}$ is the coherence length of radiation (here $\tau_{c}$ is the coherence time and $c$ is the speed of light). Radiation emitted along the optical path $L$ is measured at the reference point $\vec{X}_{0}, L_{0}$ is the optical path from the trajectory to the detector when the particle is at the maximum (or minimum) elongation point and $t, t_{0}$ are the emission times (see ref. [12]). The limited coherence length of betatron radiation, given by a large strength parameter $K=\gamma \psi_{0}>1\left(\psi_{0}\right.$ is the maximum deflection 
angle of the trajectory with respect to the $z$-axis) generally ensures that $\Delta L>L_{c}$.

In this work we provide a substantial improvement of the method discussed above by means of the real analysis of $\gamma_{c}$, thus extending the applicability to radiation sources characterized by $K<1$. Our interest is in particular the detection of the beam properties in undulators with low $K$. The paper is organized as follows. In the next section we describe a two-particle model to introduce the benefits of the analysis of $\operatorname{Re} \gamma_{c}$ with respect to $\left|\gamma_{c}\right|$. In the third section we show and discuss the results of a 2-dimensional Monte Carlo simulation with an ensemble of $10^{4}$ particles. Here a 2-period undulator which matches the parameters of the superconducting undulator of the LHC synchrotron light monitor at CERN is considered. Transverse anisotropies of protons distribution can be effectively resolved with the improved method. Finally we collect our conclusions in the last section.

Two-particle model. - We model a plane undulator with harmonic field $\vec{B}(x, 0, z)=\left[0, B_{0} \sin k_{u} z, 0\right]$ of period length $\lambda_{u}=28 \mathrm{~cm}$, field strength $B_{0}=5 \mathrm{~T}$ and periods $N_{u}=2$. Notice that $\vec{B}$ is considered only as a function of $z$, and the field dependence in the transverse directions is neglected on the scale of the proton beam size. In the weak undulator condition $K<1$ with $\dot{z} \approx \beta c$ the particle trajectory is given by $x(z)=\frac{K}{\beta \gamma k_{u}} \sin \left(k_{u} z\right)$ [15], where $k_{u}=2 \pi / \lambda_{u}$. The field $\vec{E} \cdot \overrightarrow{u_{x}}$ detected at $z=z_{0}$ is given using the Liénard-Wiechert formula at the retarded time by

$$
\vec{E} \cdot \overrightarrow{u_{x}}=\frac{e}{c k_{o}^{3} R}\left[\dot{\beta}_{x} \sin \alpha^{\prime}-\beta_{z}^{3} k_{u}^{2} c a \sin \left(k_{u} z\right)\right]
$$

where $k_{o}=1-\vec{\beta} \cdot \vec{n}, \vec{n}$ is a unit vector directed from the position of the charge towards the observation point, $\alpha^{\prime}$ is the angle between $\vec{n}$ and $\overrightarrow{\dot{\beta}}, a=K /\left(\beta \gamma k_{u}\right), e$ is the elementary charge and the approximation of small angles of observation is used $\left(\vec{n} \cdot \overrightarrow{u_{z}} \approx 1\right)$.

Assuming now that two particles have the axis of average motion with small displacements $\pm x_{d}$ with respect to the undulator axis, the phase of the radiation from each particle at the detector has a minimum close to $\left(z_{0}, \pm x_{d}\right)$ (see fig. 1). Since the maximum angle, $\psi_{0}<1 / \gamma$, of the trajectory in a weak undulator is smaller than the natural radiation opening angle, the wavefront properties of the radiation emitted instantaneously due to a transverse acceleration can be observed. Taking a reference point $x_{0}$ far away from the undulator axis we expect two solutions, $\phi\left(x_{0}\right)=\phi\left(x_{1}\right)$ and $\phi\left(x_{0}\right)=\phi\left(x_{2}\right)$, of equal phase.

Here below we show that the analysis of the real part of $\gamma_{c}$ allows the estimation of the positions $x_{1}$ and $x_{2}$, i.e. the distance $2 x_{d} \approx\left|x_{1}-x_{2}\right| / 2$ between the two particles. The initial positions of particles are $x_{1 i}=200 \mu \mathrm{m}, z_{1 i}=$ $0 \mu \mathrm{m}$ and $x_{2 i}=-200 \mu \mathrm{m}, z_{2 i}=5 \mu \mathrm{m}$. The detector is at $z_{0}=12 \mathrm{~m}$ and the reference point at $x_{0}=-4.6 \mathrm{~mm}$. The measure of the real part can be performed exploiting a modified Young interferometer [14] in which the intensity

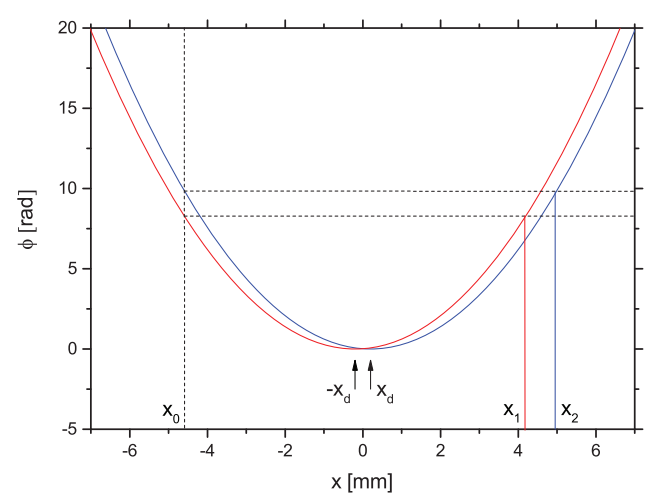

Fig. 1: (Colour online) Phase of the wavefronts detected on a transverse plane at a distance of $12 \mathrm{~m}$ from the radiation source. The radiation sources are two ultra-relativistic particles with initial positions $x_{1 i}=200 \mu \mathrm{m}$ (blue curve) and $x_{2 i}=-200 \mu \mathrm{m}$ (red curve). $x_{1}, x_{2}$ are the solutions of equal phase $\phi\left(x_{0}\right)=$ $\phi\left(x_{1}\right)$ and $\phi\left(x_{0}\right)=\phi\left(x_{2}\right)$. The minima of the red and blue functions are at $\pm x_{d}$.

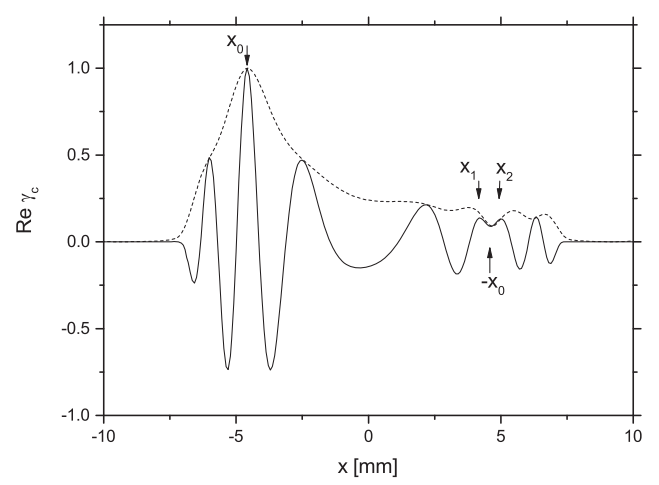

Fig. 2: Real part (solid line) and modulus (dashed line) of the coherence factor $\gamma_{c}$ of radiation emitted from two particles at a distance $12 \mathrm{~m}$ from detector. Solutions $x_{1}$ and $x_{2}$ coincide with the peak positions of $\operatorname{Re} \gamma_{c}$.

of the interference pattern is measured at the center of two slits on the detection plane. The first slit is fixed at the point $\vec{X}_{0}$, the other slit is moved transversally to the optical axis. The real part of $\gamma_{c}$ at the lab frame is shown in fig. 2, compared to $\left|\gamma_{c}\right|$ (dashed line). It is evident that the displacement between particles is given only taking $\operatorname{Re} \gamma_{c}$; arrows in fig. 2 are the positions $x_{1}$ and $x_{2}$ corresponding to the relative maxima of $\operatorname{Re} \gamma_{c}$ due to the condition of equal phase discussed above, while taking $\left|\gamma_{c}\right|$ we cannot recognize the positions of $x_{1}$ and $x_{2}$ despite the local minimum at $x=4.6 \mathrm{~mm}$. One can wonder if such a behavior described by the model can be extended to an ensemble of particles in order to resolve the transverse non-uniformity of the beam. This is answered through the simulations presented below.

\section{Simulation and results. -}

Resolving power. A Monte Carlo simulation was performed with an ensemble of particles with transverse 


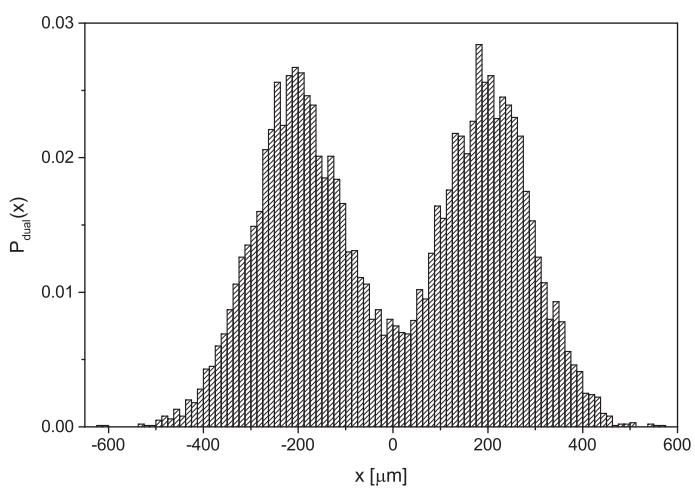

Fig. 3: Distribution of $10^{4}$ protons on the transverse axis $x$. The partly hollow center can be detected with the real analysis of $\gamma_{c}$.

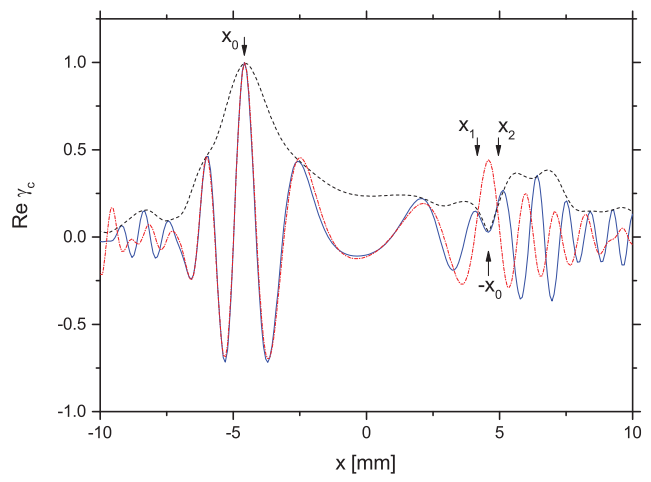

Fig. 4: (Colour online) $\operatorname{Re} \gamma_{c}$ of an ensemble of protons (blue curve) transversally distributed as in fig. 3 . The reference point is $x_{0}=-4.6 \mathrm{~mm} . x_{1}$ and $x_{2}$ are the solutions foreseen by the two-particle model. Results are compared with $\left|\gamma_{c}\right|$ (dashed line). The red dash-dotted curve is $\operatorname{Re} \gamma_{c}$ of radiation emitted by a normal distribution of protons.

initial distribution $P_{\text {dual }}(x)=\frac{1}{\sigma \sqrt{2 \pi}}\left[e^{-\frac{\left(x-\mu_{1}\right)^{2}}{2 \sigma^{2}}}+e^{-\frac{\left(x-\mu_{2}\right)^{2}}{2 \sigma^{2}}}\right]$. The two normal distributions in $P_{\text {dual }}(x)$ have $\sigma=100 \mu \mathrm{m}$ and mean values $\mu_{1}=200 \mu \mathrm{m}, \mu_{2}=-200 \mu \mathrm{m}$, thus a central hollow decrease with a full-width half-maximum of about $100 \mu \mathrm{m}$ is present as shown in fig. 3. The initial positions of particles along $z$ was uniformly distributed in the range from $-30 \mu \mathrm{m}$ to $30 \mu \mathrm{m}$. This range ensures that the bunch length is greater than the coherence length, i.e., an adjacent slice would be temporally uncoupled with the simulated slice. The others relevant parameters used in the simulation are: beam energy $=450 \mathrm{GeV}$, $\gamma=480 K=7.12 \cdot 10^{-2}, \lambda=610 \mathrm{~nm}$ and the detection distance from the source is $12 \mathrm{~m}$. The $450 \mathrm{GeV}$ beam energy at the injection ensures that the power spectral density of the radiation produced by the dipole at the entrance of the undulator is negligible at the wavelengths of the undulator radiation and no other source can affect the radiation at the exit of the undulator. Results about the real part of $\gamma_{c}$ are shown in fig. 4 for a fixed point $x_{0}=-4.6 \mathrm{~mm}$. Dashed line (black) and solid line (blue) are shown to compare the results to the case of

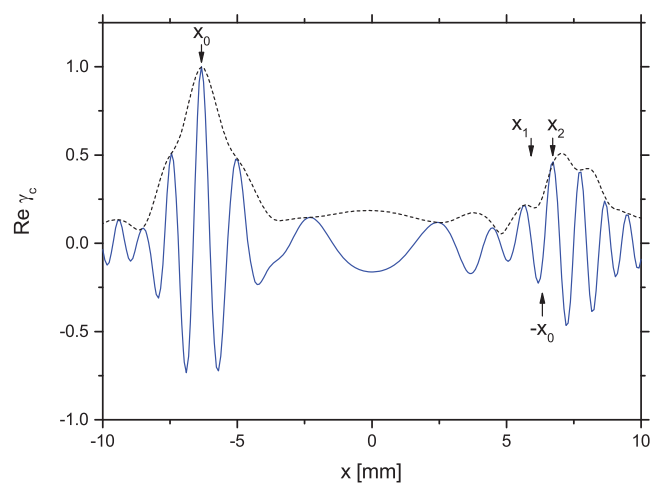

Fig. 5: (Colour online) Real part of $\gamma_{c}$ with $x_{0}=-6.3 \mathrm{~mm}$. Note that the resolution between $x_{1}$ and $x_{2}$ is improved with respect to $\operatorname{Re} \gamma_{c}$ at $x_{0}=-4.6 \mathrm{~mm}$ (see fig. 4). The dashed line is $\left|\gamma_{c}\right|$.

$\left|\gamma_{c}\right|$ and $\operatorname{Re} \gamma_{c}$, respectively. The effect of the source non-uniformity is clearly observable at $x=-x_{0}$. The maximum of coherence is split into two additional peaks. Arrows are the peak positions foreseen by the model by imposing $x_{1 i}=\mu_{1}$ and $x_{2 i}=\mu_{2}$. Although the spatial coherence of radiation is strongly reduced by the extended source, the solutions $x_{1}, x_{2}$ are in good agreement with the peak positions. On the contrary, the modulus of $\gamma_{c}$ (dashed line) cannot be easily used to recognize the peak positions of the proton transverse distribution. In order to show that the two additional peaks at $x \approx x_{1}$ and $x \approx x_{2}$ are originated by the non-uniformity of the source, we performed a simulation by using a Gaussian distribution $P_{\text {single }}(x)=\frac{1}{\sigma^{\prime} \sqrt{2 \pi}} e^{-\frac{x^{2}}{2 \sigma^{\prime 2}}}\left(\sigma^{\prime}=200 \mu \mathrm{m}\right)$ instead of $P_{\text {dual }}(x)$. Results are shown in fig. 4 (red dash-dotted line). As expected the two additional peaks disappear.

Peaks resolution can be substantially increased by increasing the distance of the reference point from axis $z$. This is shown in fig. 5 where we used a reference point $x_{0}=-6.3 \mathrm{~mm}$ further away from the $z$-axis. On the contrary the modulus of $\gamma_{c}$ does not improve the resolving power (see dashed line).

Deduction of the source size. As discussed in ref. [12] the asymmetric lateral coherence applied to betatron radiation can be used to know the source size of the particle beam. The features of the lateral maxima are easily related to the transverse size. We investigate here how the source size of the particle beam affects the maximum of the asymmetric lateral coherence of the undulator radiation in the Fraunhofer limit at low $K$. The distance from the source to the detector was increased with respect to the previous case to $28.5 \mathrm{~m}$.

The simulation was performed with an ensemble of particles with transverse initial distribution $P_{\text {beam }}(x)=$ $\frac{1}{\sigma_{b} \sqrt{2 \pi}} e^{-\frac{x^{2}}{2 \sigma_{b}^{2}}}$ and with $\sigma_{b}$ ranging from $100 \mu \mathrm{m}$ to $400 \mu \mathrm{m}$. The initial position of particles along $z$ was uniformly distributed in the range from $-30 \mu \mathrm{m}$ to $30 \mu \mathrm{m}$ (as in the previous case). 


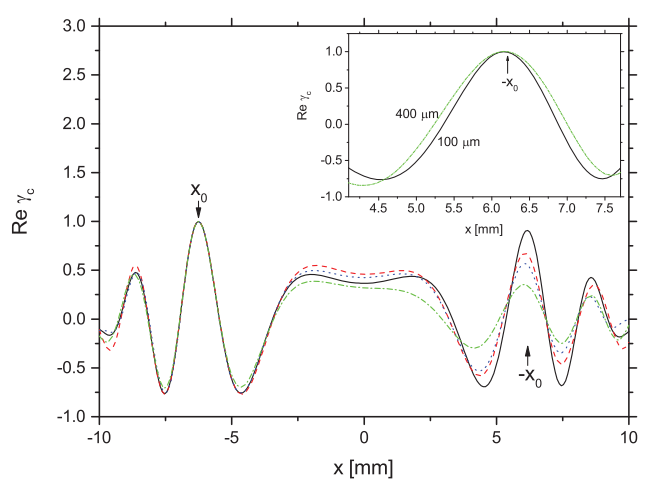

Fig. 6: (Colour online) Real parts of $\gamma_{c}$ for the normal distribution $P_{\text {beam }}(x)$ with $\sigma_{b}=100 \mu \mathrm{m}$ (black solid line), $\sigma_{b}=200 \mu \mathrm{m}$ (red dashed line), $\sigma_{b}=300 \mu \mathrm{m}$ (blue dotted line), $\sigma_{b}=400 \mu \mathrm{m}$ (green dash-dotted line). The fixed point and the detection distance were $x_{0}=-6.2 \mathrm{~mm}$ and $z_{0}=28.5 \mathrm{~m}$, respectively. The inset shows the normalized peaks for $\sigma_{b}=100 \mu \mathrm{m}$ and $\sigma_{b}=400 \mu \mathrm{m}$. Note that the peak at $-x_{0}$ of the more extensive source $\left(\sigma_{b}=400 \mu \mathrm{m}\right)$ is larger.

The source size can be effectively deduced with the amplitude of $\operatorname{Re} \gamma_{c}$ as shown in fig. 6 . Here the value of the maximum at $-x_{0}=6.25 \mathrm{~mm}$ decreases as the beam size increases demonstrating that the method is sensitive to the transverse beam size of the source. The broadband behavior of the radiation used to characterize the asymmetric lateral coherence introduces two advantages. The first advantage is the substantial increase of the measurement sensitivity with respect to a measurement with a quasi-monochromatic radiation (the monochromator reduces by many times the intensity at the detector). The second advantage is related to the shape of $\operatorname{Re} \gamma_{c}$ around $-x_{0}$. As shown in fig. 6 (see inset) the size of the beam source affects the width of the oscillation (symmetric to $x_{0}$ ), which increases with increasing size (this result confirms observations similar to those discussed in ref. [12] with betatron radiation). The increase of the width is a peculiar behavior of this method that is more evident when the measurement is performed at lower distances.

Conclusions. - We have shown that the real analysis of the asymmetric lateral coherence of radiation emitted by ultra-relativistic protons gives a substantial improvement of the resolving power with respect to the analysis of the modulus of the coherence factor.

A simplified two-particle model shows that the reciprocal distance between particles can be directly measured from the oscillating behavior of the $\operatorname{Re} \gamma_{c}$, thus a separation of $400 \mu \mathrm{m}$ was well recognized at a distance of $12 \mathrm{~m}$ from the source.

We have shown that the benefits of the method are maintained when a random (normal) distribution of emitters is considered. In fact Gaussian proton distributions with $\sigma_{b}$ between $100 \mu \mathrm{m}$ and $400 \mu \mathrm{m}$ were proven to affect the height of the symmetric peak, and could be quantitatively measured after a proper calibration.
This approach gives also additional benefits when compared with the method based on the measurement of the visibility of fringes in a classical double-slit interferometer. Exploiting the limited coherence length of radiation, both amplitude and width of the additional maxima (symmetric to the fixed point) can be used to measure the source size. The emittance and energy spread of a real beam can change the width and amplitude of the additional maxima due to the increasing of the average wavelength of the broad-spectrum radiation [16]. A quantitative estimation comes from the beam energy where a reduction of about $10^{-4}$ (in agreement with the relative energy spread at $\mathrm{LHC}$ ) introduces variations less than $0.03 \%$ in width and $0.004 \%$ in amplitude of the peak at $-x_{0}$. However, such small variations do not represent a real limitation for the applicability of the method for recovering information about the beam size.

The method can be advantageously used to perform beam size measurements in far field using broadband radiation in order to increase the instrument sensitivity (a monochromator is not needed).

$$
* * *
$$

We thank the reviewer for his/her comments. This work was supported by the Italian Ministry for University and Research (MIUR) "FIRB 2012" funds (grant No. RBFR12NK5K).

\section{REFERENCES}

[1] Phuoc K. T., Corde S., Shah R., Albert F., Fitour R., Rousseau J. P., Burgy F., Mercier B. and Rousse A., Phys. Rev. Lett., 97 (2006) 225002.

[2] Goodman J. W., Statistical Optics (John Wiley \& Sons) 2000.

[3] Naito T. and Mitsuhashi T., Phys. Rev. ST Accel. Beams, 9 (2006) 122802.

[4] Kohn V., Snigireva I. and Snigirev A., Phys. Rev. Lett., 85 (2000) 2745.

[5] Alaimo M. D., Anania M., Artioli M., Bacci A., Bellaveglia M., Ciocci F., Chiadroni E., Cianchi A., Dattoli G., Di Pirro G., Ferrario M., Gatti G., Giannessi L., Manfredda M., Martucci R., Mostacci A., Paroli B., Petralia A., Petrillo V., Pompili R., Potenza M. A. C., Quattormini M., Rau J., Redoglio D., Rossi A. R., Serafini L., Surrenti V., Torre A., Vaccarezza C. and Villa F., Opt. Express, 22 (2014) 30013.

[6] Siano M., Paroli B., Chiadroni E., Ferrario M. and Potenza M. A. C., Opt. Express, 23 (2015) 32888.

[7] Yabashi M., Tamasaku K. and Ishikawa T., Phys. Rev. Lett., 87 (2001) 140801.

[8] Singer A., Lorenz U., Sorgenfrei F., Gerasimova N., Gulden J., Yefanov O. M., Kurta R. P., Shabalin A., Dronyak R., Treusch R., Kocharyan V., Weckert E., Wurth W. and Vartanyants I. A., Phys. Rev. Lett., 111 (2013) 034802. 
[9] Lengeler B., Schroer C., Tummler J., Benner B., Richwin M., Snigirev A., Snigireva I. and Drakopoulos M., J. Synchrotron Radiat., 6 (1999) 1153.

[10] Cyrille T., Guenther R. and Ian M., Phys. Rev. ST Accel. Beams, 13 (2010) 022805.

[11] Born M. and Wolf E., Principles of Optics: Electromagnetic Theory of Propagation, Interference and Diffraction of Light (Cambridge University Press) 1999.

[12] Paroli B., Chiadroni E., Ferrario M., Petrillo V., Potenza M. A. C., Rossi A. R., Serafini L. and SHPAKOV V., EPL, 111 (2015) 44003.
[13] Paroli B., Chiadroni E., Ferrario M., Mostacci A., Petrillo V., Potenza M. A. C., Rossi A. R. and Serafini L., Nucl. Instrum. Methods Phys. Res. B, 355 (2015) 217.

[14] Paroli B., Chiadroni E., Ferrario M. and Potenza M. A. C., Opt. Express, 23 (2015) 29912.

[15] Hofmann A., The Physics of Synchrotron Radiation (Cambridge: Cambridge University Press) 2004.

[16] Clarke J. A., The Science and Technology of Undulators and Wigglers (Oxford University Press, Oxford) 2004 